\title{
Advanced Corneal Cross-Linking System with Fluorescence Dosimetry
}

\author{
Marc D. Friedman, Radha Pertaub, David Usher, Evan Sherr, \\ Pavel Kamaev, and David Muller
}

Research and Development, Avedro Inc., Waltham, MA 02451, USA

Correspondence should be addressed to Marc D. Friedman, mfriedman@avedro.com

Received 3 February 2012; Revised 27 April 2012; Accepted 7 May 2012

Academic Editor: David A. Wilkie

Copyright (C) 2012 Marc D. Friedman et al. This is an open access article distributed under the Creative Commons Attribution License, which permits unrestricted use, distribution, and reproduction in any medium, provided the original work is properly cited.

\begin{abstract}
Purpose. This paper describes an advanced system that combines corneal cross-linking with riboflavin with fluorescence dosimetry, the ability to measure riboflavin diffusion within the cornea both before and during UVA treatment. Methods and Results. A corneal cross-linking system utilizing a digital micromirror device (DMD) was assembled and used to measure diffusion coefficients of $0.1 \%$ riboflavin in $20 \%$ dextran in porcine eyes. A value of $(3.3 \pm 0.2) \times 10^{-7} \mathrm{~cm}^{2} / \mathrm{s}$ was obtained for the stroma. Diffusion coefficients for the transepithelial formulation of $0.1 \%$ riboflavin in $0.44 \%$ saline and $0.02 \%$ BAK were also measured to be $4.7 \pm 0.3 \times 10^{-8} \mathrm{~cm}^{2} / \mathrm{s}$ for epithelium only and $(4.6 \pm 0.4) \times 10^{-7} \mathrm{~cm}^{2} / \mathrm{s}$ for stroma only. Riboflavin consumption during a UVA treatment was also demonstrated. Conclusion. A new advanced corneal cross-linking system with fluorescence dosimetry of riboflavin has been demonstrated. It is hoped that this method may play a significant role in determining the underlying mechanisms of corneal cross-linking and assist with the development of additional riboflavin formulations. Moreover, dosimetry may prove valuable in providing a method to account for the biological differences between individuals, potentially informing cornea-specific UVA treatment doses in real time.
\end{abstract}

\section{Introduction}

Corneal cross-linking with riboflavin was first described by Spoerl et al. [1] and later used for the treatment of keratoconus [2-5]. It has become a widely used procedure. The standard corneal cross-linking Dresden Protocol is described as the instillation of $0.1 \%$ riboflavin solution in $20 \%$ dextran for 30 minutes with drops every 3-5 minutes, followed by $365 \mathrm{~nm}$ UVA illumination with $3 \mathrm{~mW} / \mathrm{cm}^{2}$ for 30 minutes for a total dose of $5.4 \mathrm{~J} / \mathrm{cm}^{2}$ with continued instillation of riboflavin drops every 5 minutes [2]. Recently, several new protocols using different riboflavin formulations (transepithelial, nondextran, and higher concentration riboflavin) and different UVA illumination irradiance levels (accelerated cross-linking) are being developed and used clinically.

In addition several new applications have emerged. These include post-LASIK regression minimization for hyperopia [6], treatment of infectious keratitis [7,8], corneal melts [9],
Acanthamoeba Cysts [10-12], and postthermal keratoplasty stabilization [13].

Dosimetry is the ability to quantitatively measure the concentration of riboflavin penetrating the cornea to a given depth as well as the amount of riboflavin being consumed during UVA illumination. As more protocols, formulations and uses for corneal cross-linking with riboflavin are developed, cross-linking systems that use dosimetry could be of significant importance. Real-time dosimetry may be used in determining the underlying mechanisms of corneal cross-linking and assist with the development of additional riboflavin formulations. It may also be used clinically to account for the biological differences between individuals, potentially informing cornea-specific UVA treatment doses with real-time feedback.

The history of dosimetry in phototherapy has concerned mostly bulk nontransparent tissues. Wilson et al. [14] described explicit dosimetry as the average measure of photosensitizer concentration in or around the target 
tissue just prior to light treatment, and the light fluence at some points within or around the target volume. He defined implicit dosimetry as the use of photosensitizer photobleaching to generate the dose metric. The cornea is unique in that it is optically transparent making it well suited for both explicit and implicit dosimetry through fluorescence detection of corneal cross-linking with riboflavin as well as riboflavin depletion by UVA.

Fluorescence detection through the use of fluorescein in ophthalmology is widely accepted as a method for determining corneal epithelial permeability. Kruijf et al. [15] described a simple method utilizing the Fluorotron Master (Coherent Radiation Inc., Palo Alto, CA, USA) fitted with a special lens (anterior segment adaptor) for detailed scanning of the anterior segment of the eye to determine corneal epithelial permeability. Joshi et al. [16], almost a decade later, used the Fluorotron Master to calculate epithelial permeability. Because of insufficient resolution, concentrations could not be estimated, and instead the total masses in the tear film and in the cornea derived from the area under the profile were used to calculate the epithelial permeability.

More recently, Søndergaard et al. [17] used confocal fluorescence microscopy to evaluate the distribution of riboflavin in the corneal stroma under varying concentrations and application time. Cui et al. [18] used an 800-nm femtosecond laser to perform two-photon fluorescence (TPF) axial scanning to measure the concentration of fluorescein and riboflavin across the central corneal depth in a freshly enucleated feline globe model.

This paper describes an advanced system that combines both corneal cross-linking with riboflavin and fluorescence dosimetry. A digital micromirror device (DMD) UVA projector and imaging setup was assembled and used to measure riboflavin diffusion coefficients of both epithelium and stroma and to measure riboflavin consumption during a UVA treatment. Future development of this system and its implications are discussed.

\section{Material and Methods}

2.1. Fluorescence Dosimetry Experiments Using a Bench Top Digital Micromirror Device [DMD] with UV Source. A bench top corneal cross-linking system using a DMD with an additional fluorescent dosimetry camera was developed. The DMD is the same technology utilized in digital projectors and has the advantage of being able to create any unique beam shape or intensity profile. The bench top setup seen in Figure 1 is comprised of an UVA LED illuminator (UV LED NCSU033B(T), Nichia Co., Japan) with a wavelength of $365 \mathrm{~nm}$. Light emitted from the illuminator (beam uniformity of $\pm 3 \%$ Root Mean Square) is directed onto a digital micro-mirror device (DMD) (Texas Instruments, Model XGA UV DMD) via a homogenizer and lenses L1 (NT49695, Edmund Optics) and L2 (NT48-285, Edmund Optics). Light from the surface of the DMD is imaged with the L2 doublet and centered onto the eye coincident with its optical axis. A 1.3 mega pixel camera (Basler acA1300-30 gm), with L3 (Computar MLM3X-MP) and a long-pass filter, F, with



FIGURE 1: Block diagram of bench top DMD system configuration.

a cut-off wavelength $420 \mathrm{~nm}$ (LP415 UV Dichroic Blocking Filter, Midwest Optical Systems), is mounted and centered at a $45^{\circ}$ angle to the apex of the eye and its cornea.

The DMD can be configured to project UVA illumination of any arbitrary pattern with 256 (8-bit) levels of intensity which is controlled by a video signal that can be updated at a rate of $60 \mathrm{~Hz}$. This allows for rapid switching between an illumination profile intended for cross-linking treatment and that of a profile intended for fluorescent dosimetry. The system can, therefore, rapidly switch modes such that dosimetry measurements can be taken at intervals prior to and during the treatment. This is illustrated in Figure 2 where an image corresponding to a projected pattern by the DMD bench top system is shown.

During fluorescent dosimetry measurement, Figure 3, one or more vertical lines are projected. The first image shows one vertical line image, the second shows four vertical line images, and the third shows seven vertical line images. Each line image shows the cross-sectional distribution of riboflavin through the cornea at its spatial location. Riboflavin distribution within the cornea can be instantly measured at various points by projecting multiple line images onto the cornea. Additionally, this method may be used to provide corneal topography of the corneal surface as well as the riboflavin distribution through the cornea since the leading edge of the fluorescent image is a measure of the cornea's curvature.

In the following experiments, the DMD was configured to project a vertical one-pixel line width (approximately $25 \mu \mathrm{m}$ ) of illumination onto the corneal surface and set to an output irradiance of $30 \mathrm{~mW} / \mathrm{cm}^{2}$.

Three separate experiments were performed. The first experiment was to measure riboflavin diffusion through the cornea without epithelium, the second experiment was to measure riboflavin diffusion through the cornea with epithelium, and the third experiment was to measure riboflavin consumption during a UVA treatment.

In experiment 1 porcine eyes $(n=3)$ were supplied 1 day after mortem by Sioux-Preme (Sioux Center, IA, USA) and pressurized to approximately $15 \mathrm{mmHg}$ with a saline column approximately $20 \mathrm{~cm}$ above the eye. Epithelial debridement 


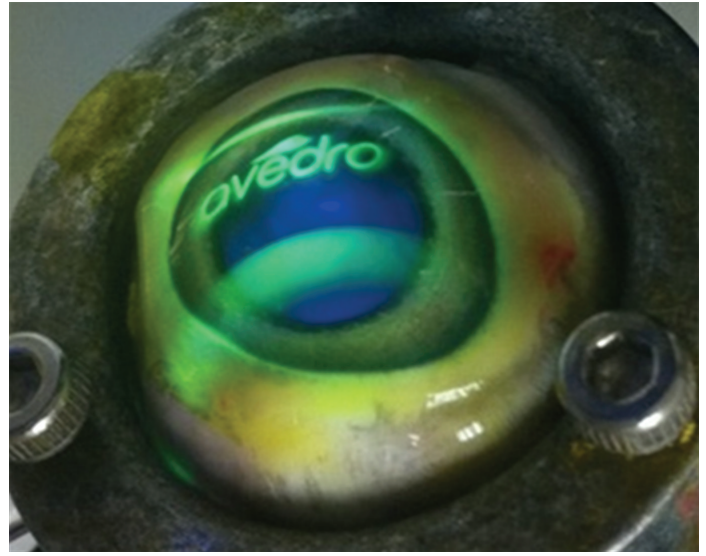

FIGURE 2: Example of the flexibility of UV source patterns projected on an excised porcine eye.

was performed with a flat blade. An image was taken prior to the application of riboflavin to serve as a baseline image. A solution of $0.1 \%$ riboflavin in $20 \%$ dextran was instilled onto the corneas every 30 seconds for 3 minutes. The corneas were then rinsed with buffered saline, and an image was recorded. This procedure was then repeated with images recorded every 3 minutes for a total of 21 minutes. In the second experiment, the previous experimental protocol was repeated this time using porcine eyes $(n=3)$ with their epithelium intact and a transepithelial solution of $0.25 \%$ riboflavin in $0.44 \%$ saline with $0.02 \%$ benzalkonium chloride (BAK).

In the third experiment, several drops of $0.25 \%$ riboflavin in $44 \%$ saline were instilled to cover deepithelialized eyes $(n=6)$ every 30 seconds for a total of 10 minutes. The eyes were rinsed with saline. Images were then recorded for each of the eyes as a baseline image. UVA treatment of eyes $(n=3)$ was placed under a uniform $10 \mathrm{~mm}$ diameter UVA beam with a wavelength of $365 \mathrm{~nm}$ at an irradiance of $30 \mathrm{~mW} / \mathrm{cm}^{2}$. After one minute the UVA treatment was temporarily stopped, and fluorescence dosimetry images were taken. This was repeated 5 more times to obtain images up to a total UVA dose of $10.8 \mathrm{~J} / \mathrm{cm}^{2}$. A set of control eyes $(n=3)$ underwent the same procedure but without UVA irradiation. Particular care was taken to keep the intervals between UVA irradiation and imaging constant.

2.2. Franz Cell Experiments. Porcine eyes were supplied 1 day after mortem by Sioux-Preme (Sioux Center, IA, USA) and were warmed to room temperature and allowed to fully swell. An automated Hansatome microkeratome (model HT 230, Chiron Vision, Hansa Research \& Development, Inc., Miami, FL) was used to create corneal flaps by excision. The average thickness of the flaps was approximately $100 \mu \mathrm{m}$ as measured with an ultrasonic pachymeter (DGH-550 Pachette 2, DGH Technology, Exton, PA, USA).

The diffusivity of riboflavin through cornea was measured by experimentation using a temperature controlled $\left(37^{\circ} \mathrm{C}\right)$ Franz cell (PermeGear, Inc. Hellertown, PA, USA). Corneal flaps were placed between the receiver and donor compartments of the Franz cells. Receiver compartments were filled with saline, and donor compartments were filled with a riboflavin solution for each formulation tested. Corneal flaps $(n=3)$ for each formulation were tested. The amount of riboflavin passing through the corneal flaps per time was measured by means of spectrophotometry, and diffusivity of riboflavin was calculated using Fick's first law.

2.3. Image Analysis. Images were exported and postanalyzed using ImageJ software (http://rsbweb.nih.gov/ij/). Crosssectional intensity profiles were plotted for each image for both before and after the addition of riboflavin. Control images of the cornea before instillation of riboflavin provided baseline intensities that were subtracted from the intensities of the cornea with riboflavin.

A cosine correction factor was used to adjust for the $45^{\circ}$ angle of incidence in order to obtain the correct corneal depth measurement. Calibration tests performed measured a resolution of $12.2 \mu \mathrm{m}$ per pixel. The resulting intensity profiles of riboflavin in the corneas were then fitted using the solution to Fick's second law of diffusion equation

$$
C=C_{0}\left(1-\operatorname{erf}\left(\frac{x}{2 \sqrt{D t}}\right)\right)
$$

where $C$ is concentration in the medium diffused, $C_{0}$ is initial concentration at boundary, $x$ is spatial distance, $D$ is diffusion coefficient, and $t$ is the time needed for diffusion.

Since riboflavin fluoresces linearly with concentration, (1) becomes as follows:

$$
I=I_{0}\left(1-\operatorname{erf}\left(\frac{x}{2 \sqrt{D t}}\right)\right),
$$

where $I$ is intensity in the cross-section of the cornea, and $I_{0}$ is initial intensity at the boundary.

\section{Results}

3.1. DMD Bench Top Experiments. For the experiment with riboflavin in dextran and no epithelium, a representative analysis can be seen in Figure 4. The raw image of the slit projection can be seen together with the extracted intensity profile. The corresponding model fit using (2) is also shown. An average diffusion coefficient of $(3.3 \pm 0.2) \times 10^{-7} \mathrm{~cm}^{2} / \mathrm{s}$ was recorded for this formulation of riboflavin. This average was calculated using all images recorded for soak times from 6 minutes through 21 minutes for all three eyes. This value compares well with the value of $(3.8 \pm 0.8) \times 10^{-7} \mathrm{~cm}^{2} / \mathrm{s}$ obtained using the Franz cell approach.

For the experiment with riboflavin in BAK solution and epithelium intact, a representative analysis can be seen in Figure 5. The analysis of the average value of the diffusion coefficient was performed for soak times 6 minutes through 21 minutes for all three eyes. In this experiment, the riboflavin diffuses through both the epithelium and the stroma, each having its own diffusion coefficient. Therefore, the curve fit was done in two parts: coefficients $C_{1}$ and $D_{1}$ for boundary intensities at the top of the epithelium and a diffusion coefficient in epithelium alone (for $x$ between 0 and 


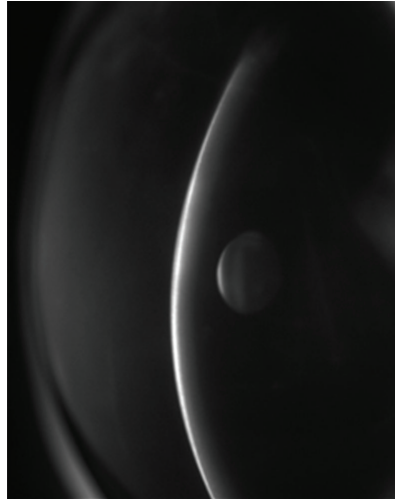

(a)



(b)

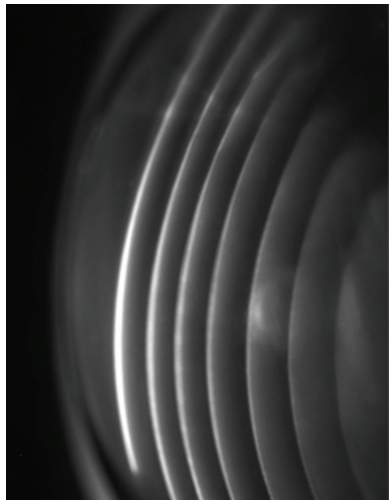

(c)

FIgURE 3: Example images of a fluorescing porcine cornea. Three images of projected patterns by the DMD bench top system (a) a vertical slit, (b) 4 parallel vertical slits, and (c) 7 parallel vertical slits.


Figure 4: The image on the left shows the intensity profile after 18 minutes of presoak with $0.1 \%$ riboflavin in $20 \%$ dextran. The corresponding plot on the right shows the intensity versus the corneal depth (blue marker) and the curve fit to the experimental data (red line).

$75 \mu \mathrm{m})$ and coefficients $C_{2}$ and $D_{2}$ for boundary intensity at the top of the stroma and a diffusion coefficient in stroma alone, respectively (for $x>75 \mu \mathrm{m}$ ).

A diffusion coefficient of $(4.7 \pm 0.3) \times 10^{-8} \mathrm{~cm}^{2} / \mathrm{s}$ was obtained for the epithelium only and a diffusion coefficient of $(4.6 \pm 0.4) \times 10^{-7} \mathrm{~cm}^{2} / \mathrm{s}$ for the stroma only for the fluorescent imaging. These values are slightly lower than the values obtained using the Franz cell method, $(2.4 \pm 0.3) \times$ $10^{-7} \mathrm{~cm}^{2} / \mathrm{s}$ for the epithelium and stroma combined and (6.5 $\pm 0.8) \times 10^{-7} \mathrm{~cm}^{2} / \mathrm{s}$ stroma only (Figure 6).

For the third experiment, the percentage drop in fluorescence intensity detected in the top 12 microns of the cornea is seen in Figure 7 with respect to the controls.

\section{Discussion}

The fluorescence dosimetry experiment using the bench top DMD with UVA source demonstrated that the value of diffusivity without epithelium compared well with those obtained with the Franz cell experiment. For the transepithelial formulation in the second experiment, the values obtained using the Franz cell for the diffusion of riboflavin with epithelium are an effective diffusion coefficient of combined epithelium and stroma. The Franz cell method is not able to deconvolve the two diffusion coefficients separately where fluorescence imaging can. The clinical implication of this finding is that fluorescence dosimetry may also be able to differentiate and account for variable epithelial hypoplasia and hyperplasia across the cornea, a very common condition in keratoconus.

The third experiment demonstrates that photo-dissociation of riboflavin over time can be detected and that, without the addition of riboflavin, the riboflavin slowly continues to diffuse into the cornea.

The current work assumes no scattering. This is an approximation as there is light scattering in the cornea at $370 \mathrm{~nm}$. Second-order enhancements are planned which will incorporate light scattering by the cornea into our image analysis models. This modeling will also account for the UVA absorbed as well as the fluorescence produced to determine the dose delivered and how this equates to the amount of corneal cross-linking produced. Temperature effects, increased depth of focus, higher resolution, calibration methods, automated alignment including automatic $x$, 

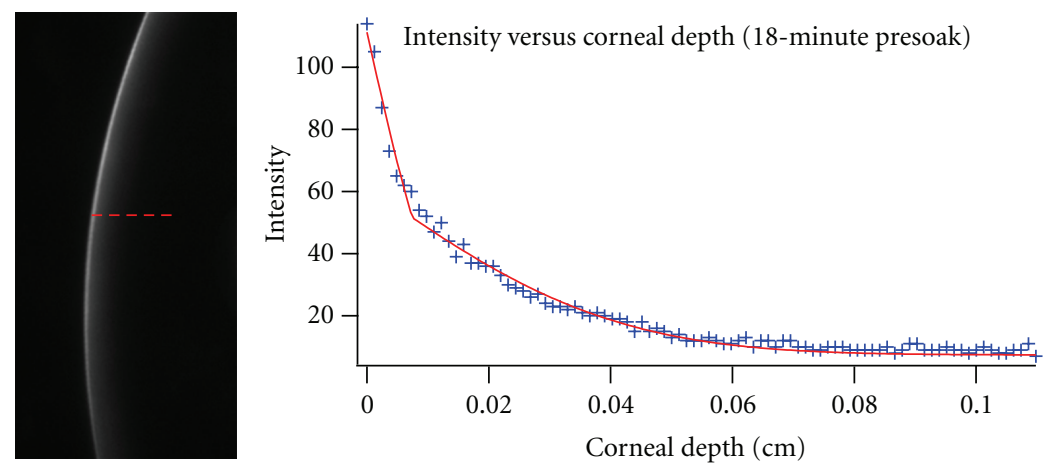

Figure 5: The image on the left shows the intensity profile after 18 minutes of presoak with $0.1 \%$ riboflavin in $0.44 \%$ saline, $0.02 \%$ BAK. The corresponding plot on the right shows the intensity versus the corneal depth (blue marker) and the biphasic curve fit to the experimental data (red line).

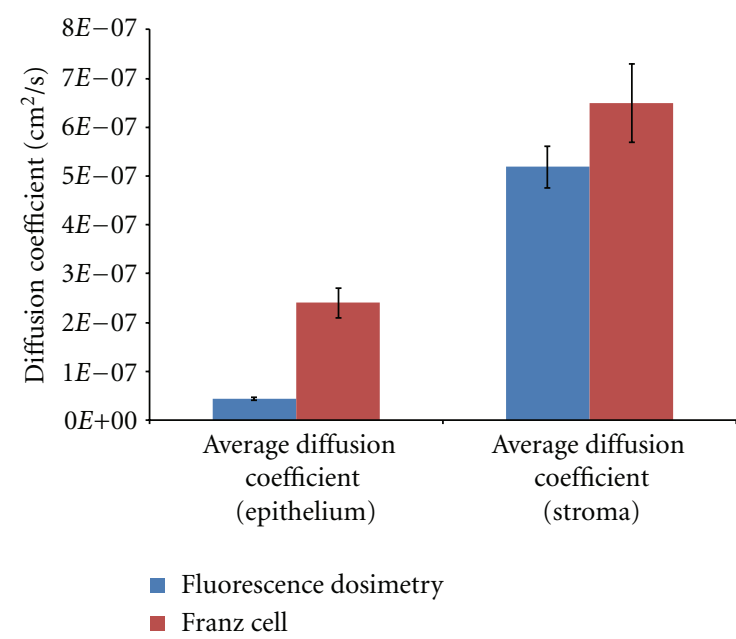

FIGURE 6: Comparison between diffusion coefficients obtained with fluorescence imaging versus a Franz cell for a $0.1 \%$ riboflavin in $0.44 \%$ saline, $0.02 \%$ BAK formulation with and without epithelium.

$y$, and $z$ fine alignment, cyclotorsion compensation based on eye, and landmark image analysis are all being investigated in an effort to produce a system with more consistent results.

This advanced system has several unique features which lend itself to possible real-time measurement in a combination UVA treatment and fluorescence-monitoring system. Beam uniformity and irradiance calibration at the corneal plane are simplified utilizing a DMD since each individual micromirrors average output power is adjustable. The system may also be used for customized corneal cross-linking procedures where the DMD patterning and pretreatment plan is based on corneal topography and or optical coherence tomography. Variables such as corneal elevations, power maps, $k$ readings, corneal thickness maps, and epithelial thickness maps may all be utilized to create an optimized cross-linking pretreatment plan and procedure. Finally, during UV treatment, this treatment plan may also respond and compensate to live dosimetry readings as riboflavin is consumed and measured at various depths. We are in the process of investigating these possibilities.
Drop in fluorescence intensity with and without



FIgURE 7: Drop in fluorescence intensity of the top 12 microns of the cornea with and without UVA dose.

\section{Conclusion}

A new advanced corneal cross-linking system with fluorescence dosimetry with riboflavin has been demonstrated. It is hoped that this method may someday play a significant role in determining the underlying mechanisms of corneal cross-linking and assist with the development of additional riboflavin formulations. Moreover, dosimetry may prove valuable in providing a method to account for the biological differences between individuals, potentially informing cornea specific UVA treatment doses in real time.

\section{References}

[1] E. Spoerl, M. Huhle, and T. Seiler, "Induction of cross-links in corneal tissue," Experimental Eye Research, vol. 66, no. 1, pp. 97-103, 1998.

[2] G. Wollensak, E. Spörl, and T. Seiler, "Treatment of keratoconus by collagen cross linking," Der Ophthalmologe, vol. 100, no. 1, pp. 44-49, 2003. 
[3] G. Wollensak, E. Spoerl, and T. Seiler, "Riboflavin/ultravioletA-induced collagen crosslinking for the treatment of keratoconus," American Journal of Ophthalmology, vol. 135, no. 5, pp. 620-627, 2003.

[4] A. Caporossi, S. Baiocchi, C. Mazzotta, C. Traversi, and T. Caporossi, "Parasurgical therapy for keratoconus by riboflavin-ultraviolet type A rays induced cross-linking of corneal collagen: preliminary refractive results in an Italian study," Journal of Cataract and Refractive Surgery, vol. 32, no. 5, pp. 837-845, 2006.

[5] F. Raiskup-Wolf, A. Hoyer, E. Spoerl, and L. E. Pillunat, "Collagen crosslinking with riboflavin and ultraviolet-A light in keratoconus: long-term results," Journal of Cataract and Refractive Surgery, vol. 34, no. 5, pp. 796-801, 2008.

[6] J. A. Kanellopoulos, "Higher fluence CXL," in Proceedings of the 7th International Congress of Corneal Cross-Linking, Zurich, Switzerland, December 2011, Clinical Results-Section 7.

[7] S. A. R. Martins, J. C. Combs, G. Noguera et al., "Antimicrobial efficacy of riboflavin/UVA combination $(365 \mathrm{~nm})$ in vitro for bacterial and fungal isolates: a potential new treatment for infectious keratitis," Investigative Ophthalmology \& Visual Science, vol. 49, no. 8, pp. 3402-3408, 2008.

[8] H. M. Anwar, A. M. El-Danasoury, and A. N. Hashem, "Corneal collagen crosslinking in the treatment of infectious keratitis," Clinical Ophthalmology, vol. 5, no. 1, pp. 1277-1280, 2011.

[9] O. Richoz and F. Hafezi, "CXL for infectious corneal meltingan update," in Proceedings of the 7th International Congress of Corneal Cross-Linking, Zurich, Switzerland, December 2011, Clinical Results-Section 7.

[10] O. Richoz and F. Hafezi, "CXL in acanthamoeba keratitis," in Proceedings of the 7th International Congress of Corneal Cross-Linking, Zurich, Switzerland, December 2011, Clinical Results-Section 7.

[11] R. Lamy, E. Cahn, and J. M. Stewart, "Riboflavin + UVA for the inactivation of acanthamoeba cysts," in Proceedings of the 7th International Congress of Corneal Cross-Linking, Zurich, Switzerland, December 2011, Clinical Results-Section 7.

[12] Y. A. Khan, R. T. Kashiwabuchi, S. A. Martins et al., "Riboflavin and ultraviolet light a therapy as an adjuvant treatment for medically refractive acanthamoeba keratitis: report of 3 cases," Ophthalmology, vol. 118, no. 2, pp. 324-331, 2011.

[13] A. Cummings, "Standard versus high intensity CXL after thermal keratoplasty," in Proceedings of the 7th International Congress of Corneal Cross-Linking, Zurich, Switzerland, December 2011, Controversies-Section 9.

[14] B. C. Wilson, M. S. Patterson, and L. Lilge, "Implicit and explicit dosimetry in photodynamic therapy: a new paradigm," Lasers in Medical Science, vol. 12, no. 3, pp. 182-199, 1997.

[15] E. J. F. M. Kruijf, L. Laterveer, J. P. Boot, J. A. V. Best, J. A. M. Ramselaar, and J. A. Oosterhuis, "A simple method for determination of corneal epithelial permeability in humans," Current Eye Research, vol. 6, no. 11, pp. 1327-1334, 1987.

[16] A. Joshi, D. Maurice, and J. R. Paugh, "A new method for determining corneal epithelial barrier to fluorescein in humans," Investigative Ophthalmology \& Visual Science, vol. 37, no. 6, pp. 1008-1016, 1996.

[17] A. P. Søndergaard, J. Hjortdal, T. Breitenbach, and A. Ivarsen, "Corneal distribution of riboflavin prior to collagen crosslinking," Current Eye Research, vol. 35, no. 2, pp. 116-121, 2010.
[18] L. Cui, K. R. Huxlin, L. Xu, S. MacRae, and W. H. Knox, "High-resolution, noninvasive, two-photon fluorescence measurement of molecular concentrations in corneal tissue," Investigative Ophthalmology \& Visual Science, vol. 52, no. 5, pp. 2556-2564, 2011. 




The Scientific World Journal
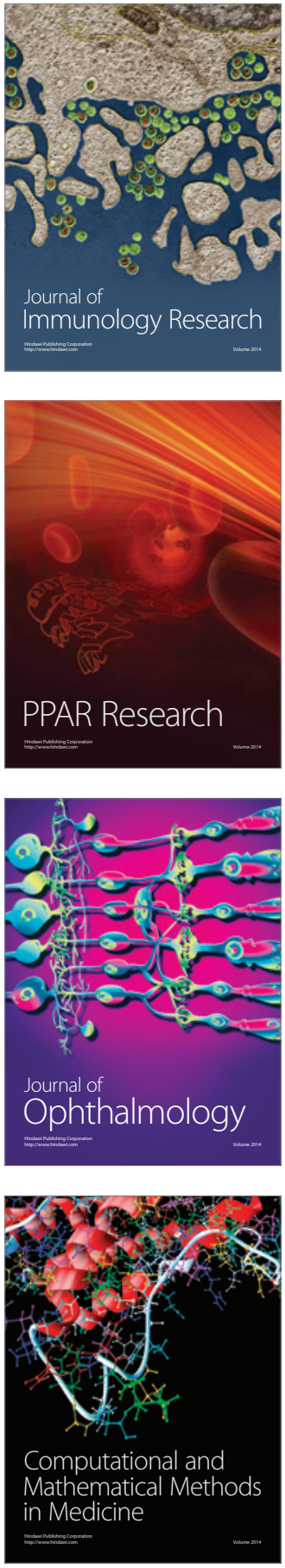



Gastroenterology

Research and Practice


\section{Hindawi}

Submit your manuscripts at

http://www.hindawi.com
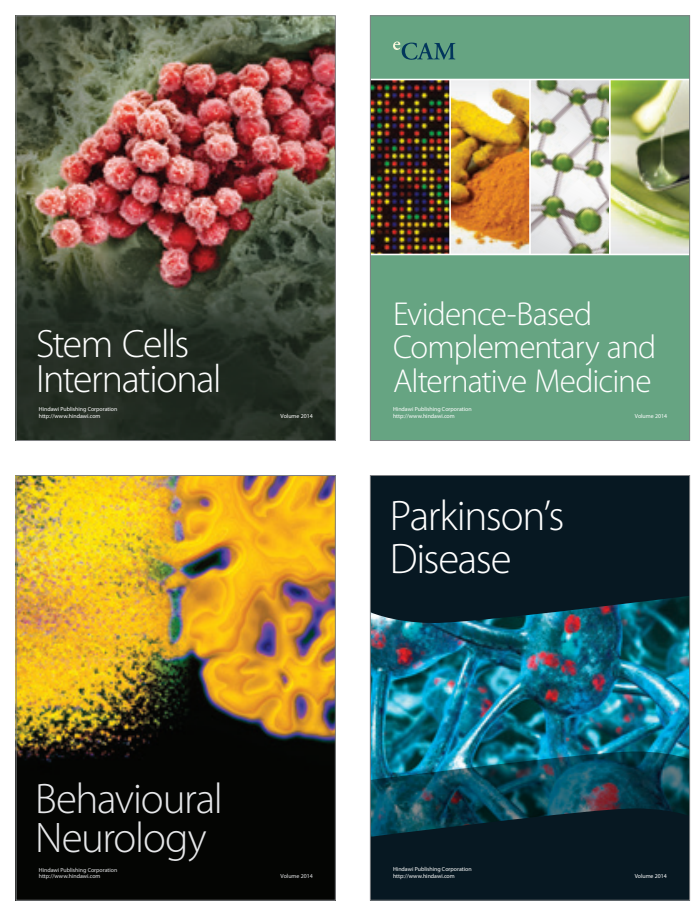



Journal of
Diabetes Research

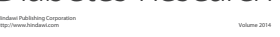

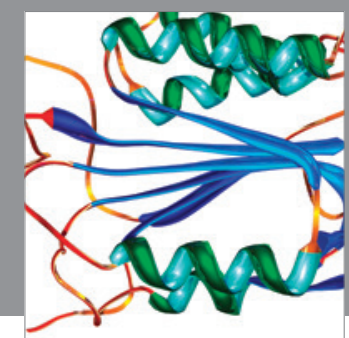

Disease Markers
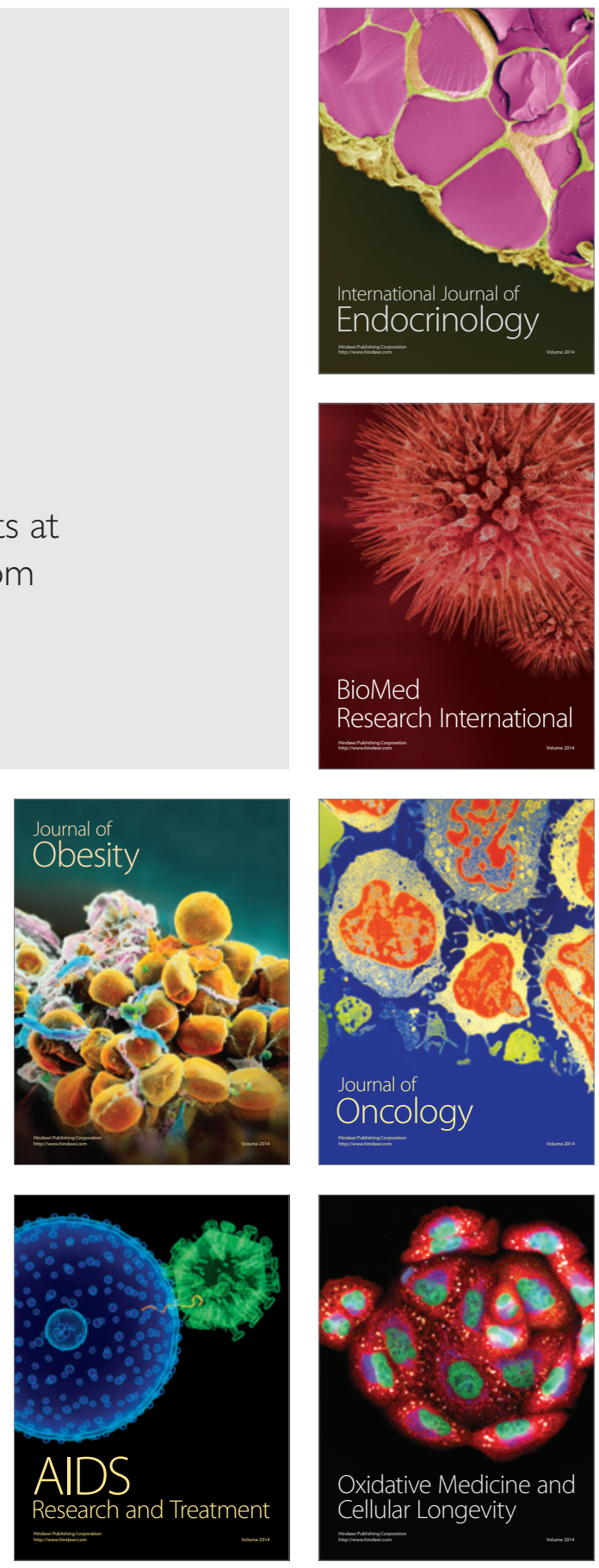